\title{
February 2018 Pulmonary Case of the Month
}

\section{Lewis J. Wesselius, MD}

\author{
Department of Pulmonary Medicine \\ Mayo Clinic Arizona \\ Scottsdale, AZ USA
}

\section{History of Present IIIness}

A 75-year-old woman was diagnosed with a thymic carcinoid tumor in April, 2015 (Figure 1).

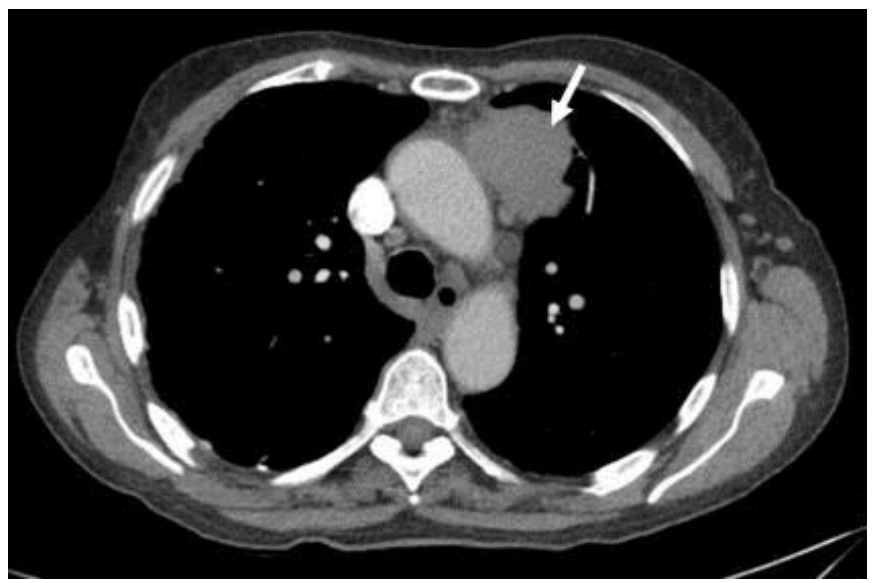

Figure 1. Representative image from the preoperative CT scan performed in April 2015 showing an anterior mediastinal mass (arrow).

This was treated with surgical resection followed by radiation therapy. She began having cough and dyspnea 1 to 2 months later and in August, 2015 had a thoracic CT scan of her chest (Figure 2).

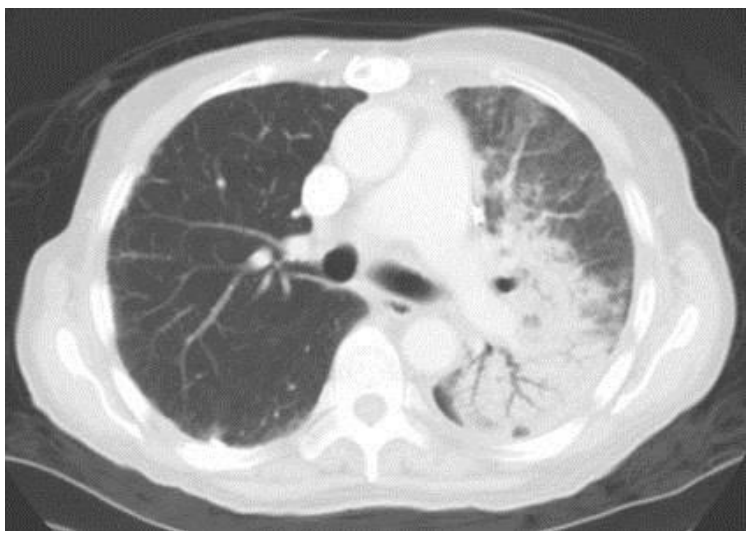

Figure 2. Representative image in lung windows from the second thoracic CT scan performed in August 2015. 
Which of the following are true?

1. Bronchoscopy should be performed

2. She should be given an empiric course of antibiotics

3. The most like diagnosis is radiation pneumonitis

4. 1 and 3

5. All of the above 


\section{Correct!}

\section{1 and 3}

The repeat thoracic CT scan shows that the mass present in Figure 1 has disappeared but there is consolidation in the left upper lobe and the superior subsegment of the left lower lobe. Her chronic symptoms of cough are nonspecific but more suggestive or radiation pneumonitis than an acute infection. Radiation pneumonitis usually occurs $2-3$ months after therapy and can be mild or severe. Symptoms include may include cough, shortness of breath, low grade fever or chest pain and can be indistinguishable from infectious pneumonitis. Although empiric antibiotics will likely do her no harm, her mild symptoms with rather marked lung consolidation is more suggestive of radiation pneumonitis than infectious pneumonia.

She underwent bronchoscopy with transbronchial biopsies which showed only "organizing pneumonitis" without organisms being seen. She was felt to most likely have radiation pneumonitis.

Which of the following is the generally recommended therapy for radiation pneumonitis?

1. Corticosteroids

2. Cyclosporine

3. Tacrolimus

4. 1 and 3

5. All of the above 


\section{Correct! \\ 1. Corticosteroids}

Corticosteroids are the usual therapy for radiation pneumonitis although other antiinflammatories have been used. There are no controlled, randomized trials on the treatment of radiation pneumonitis (1). Patients with mild symptoms who are mostly asymptomatic can be observed. For patients with more severe complaints, corticosteroids are given. The dose and duration of therapy is unclear but for most patients prednisone 30-40 mg daily for two weeks followed by a slow reduction over six to twelve weeks results in clinical improvement. A relapse may occur after discontinuation of corticosteroids. A substantial reduction in symptoms is normally seen, as well as an improvement of the radiological abnormalities.

She was felt to most likely have radiation pneumonitis and was treated with steroids for month with subjective improvement, but symptoms returned after discontinuation of steroids. A repeat thoracic CT scan was performed in October, 2016 (Figure 3).

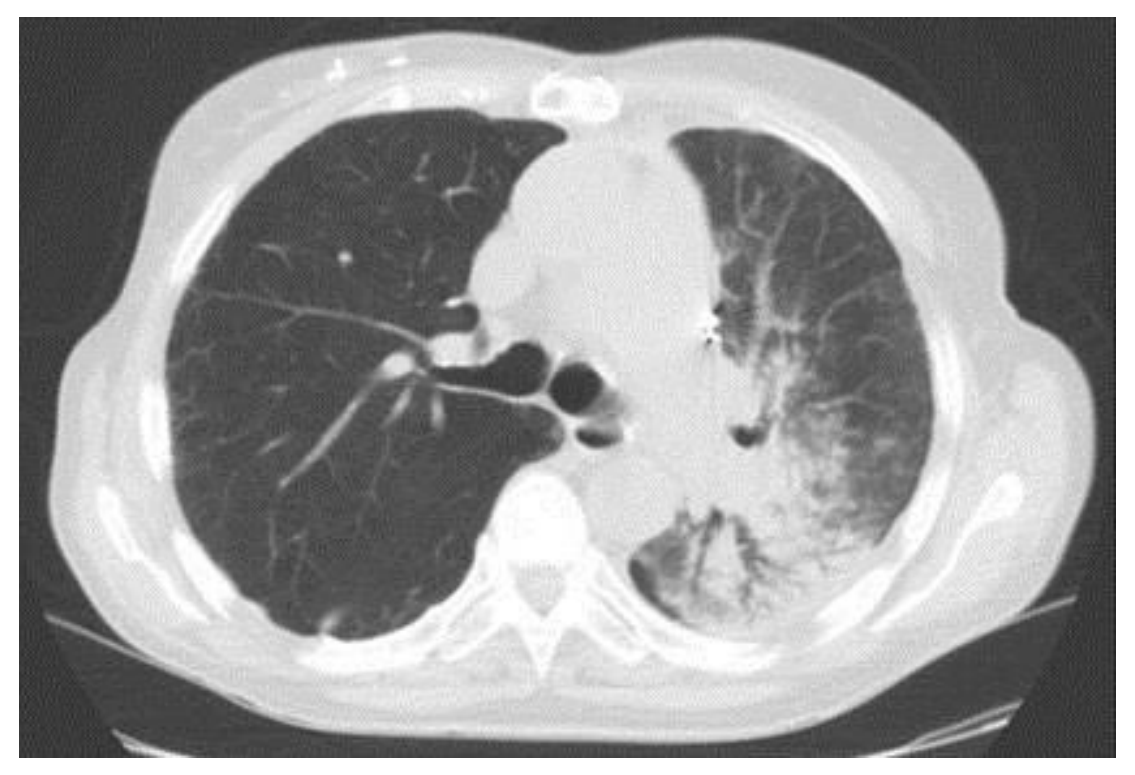

Figure 3. Representative image in lung windows from the thoracic CT scan performed in October 2015 at approximately the same level as Figure 2.

Which of the following should be done at this time?
1. Bronchoscopy
2. Treat with a course of tacrolimus
3. Treat with a repeat course of corticosteroids
4. 1 or 3
5. Any of the above 


\section{Correct!}

\section{1 or 3}

Most likely she has had a recurrence of her radiation pneumonitis when the corticosteroids were tapered. If there was a suspicion of another process bronchoscopy could be performed. Tacrolimus is generally not used for radiation pneumonitis (1). She was again started on prednisone with clinical improvement. She was tapered off prednisone in January, 2016, but her symptoms of dyspnea and cough returned. She was referred for a second opinion.

In addition to the thymic carcinoid tumor, she has a past medical paroxysmal atrial fibrillation, hypertension, and hypothyroidism. Her medications included apixaban, levothyroxine, and diltiazem. She had a minimal smoking history of 5 pack years having quit in 1974. Her physical exam showed a few crackles on right but was otherwise unremarkable. Laboratory evaluation revealed a normal complete blood count with a normal differential and an elevated C-reactive protein.

A repeat thoracic CT scan was obtained (Figure 4).

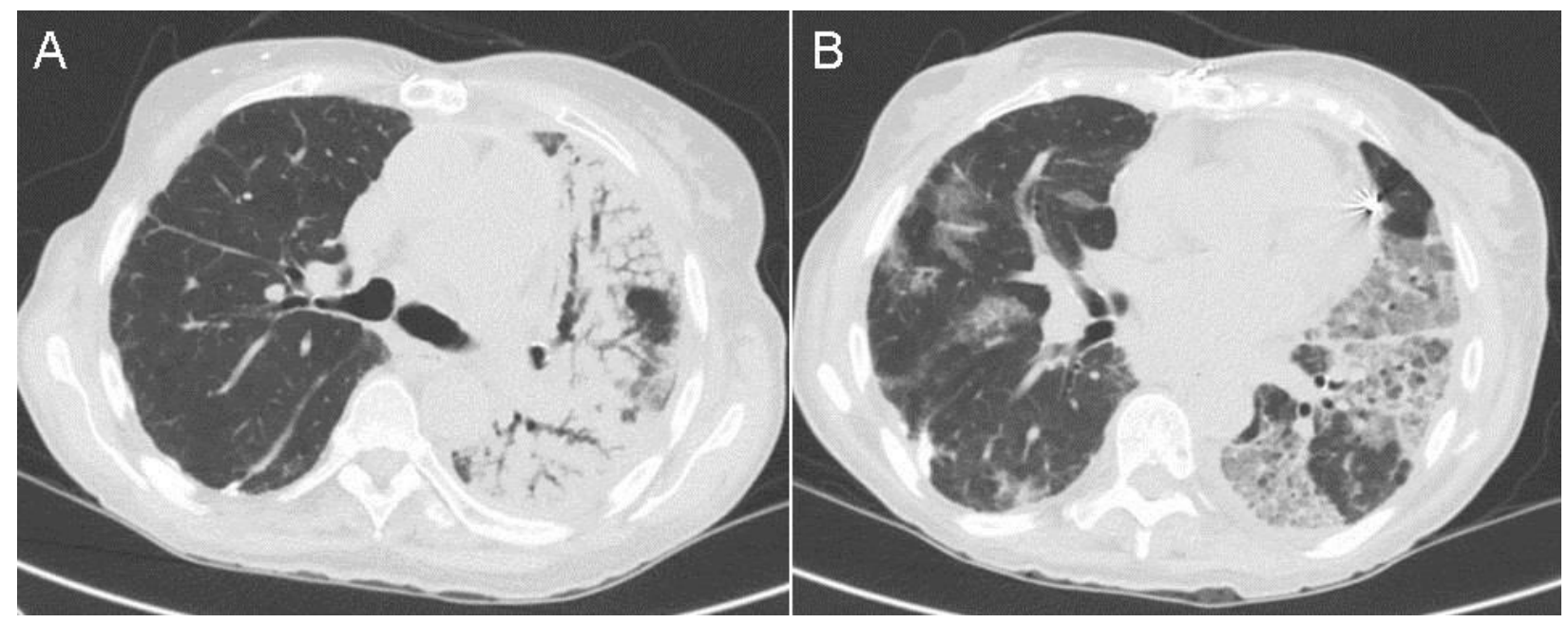

Figure 4. Two views in lung windows from the repeat thoracic CT scan.

What should be done at this time?

1. A course of cyclosporine

2. Another course of corticosteroids

3. Repeat bronchoscopy

4. 1 and 3

5. All of the above 


\section{Correct! \\ 3. Repeat bronchoscopy}

The repeat CT scan shows worsening of the consolidation in the left lung but now there is also some patchy areas of consolidation in the right lung. This is not the usual course or radiation pneumonitis. Since another diagnosis seems possible and the first transbronchial biopsy showed nonspecific changes, a repeat bronchoscopy is indicated. Another course of corticosteroids or cyclosporine might be the correct therapy but only if repeat bronchoscopy is consistent with radiation pneumonitis.

A repeat bronchoscopy with bronchoalveolar lavage and transbronchial biopsy was performed. Bronchoalveolar lavage showed $12 \%$ eosinophils. The biopsy showed subacute injury with organization and extravascular eosinophils suggestive of eosinophilic pneumonia (Figure 5).

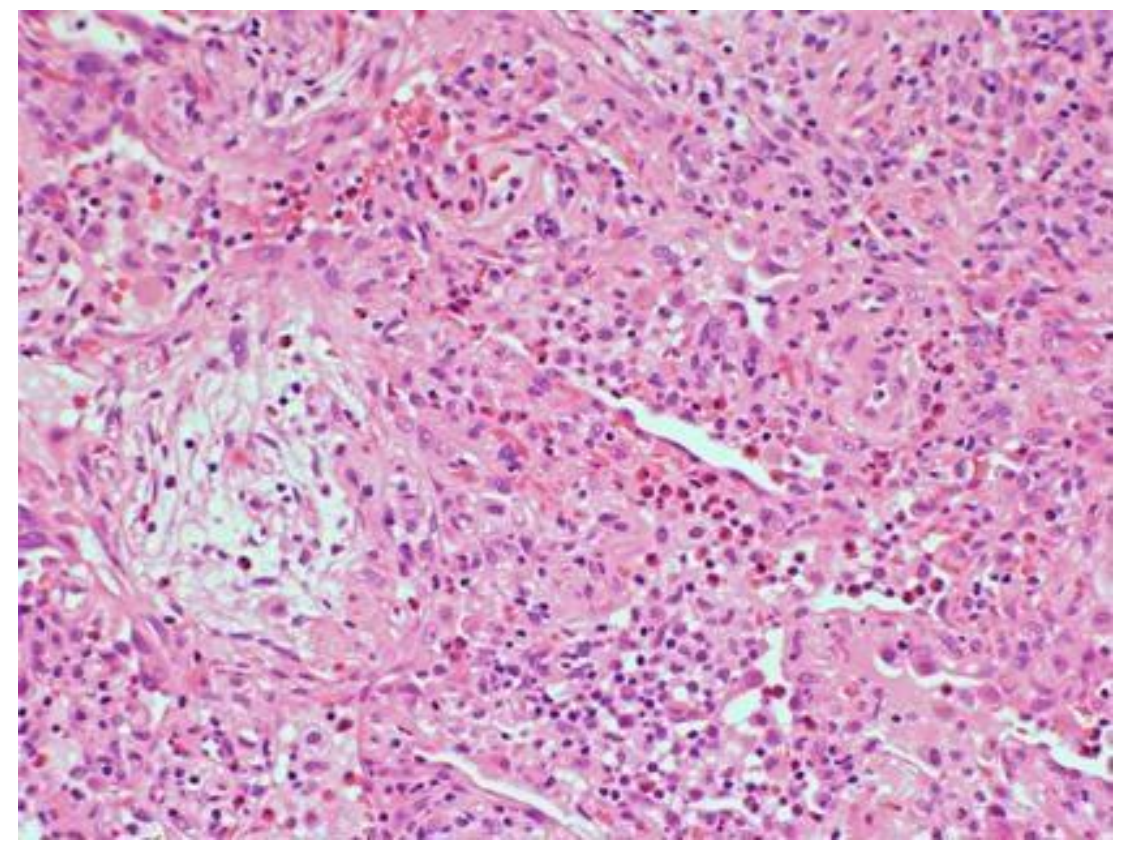

Figure 5. Transbronchial biopsy showing subacute injury with extravasation of eosinophils ( $H$ \& E staining).

A diagnosis of radiation-induced eosinophilic pneumonia was made.

What treatment should the patient receive at this time?

1. Observation

2. Repeat course of corticosteroids

3. Tacrolimus

4. 1 or 3

5. Any of the above 


\section{Correct! \\ 2. Repeat course of corticosteroids}

Our patient was restarted on steroid therapy (1 mg/kg prednisone) and has clinically improved. She has returned home and will be having repeat outside imaging.

This patient had radiographic findings that were atypical for radiation pneumonitis (significant infiltrate outside of the radiation port to the thymus, including in opposite lung). Eosinophilic pneumonia can be associated with drug therapy, exposure to toxins or can be idiopathic. It has also been reported in association with radiation therapy $(2,3)$. When associated with radiation therapy, eosinophilic pneumonia has been seen most commonly after radiation for breast cancer. Patients demonstrate alveolar infiltrates that may develop both inside and outside of the radiation port within 12 months of radiation therapy, without another identifiable cause of eosinophilic pneumonia. Patients typically respond well to corticosteroids.

\section{References}

1. Weytjens R, Erven K, De Ruysscher D. Radiation pneumonitis: occurrence, prediction, prevention and treatment. Belg J Med Oncol. 2013;7(4):105-10.

2. Solomon J, Schwartz M. Drug-, toxin-, and radiation therapy-induced eosinophilic pneumonia. Semin Respir Crit Care Med 2006;27:192-8. [CrossRef] [PubMed]

3. Cottin V, Frognier $\mathrm{R}$, Monnot $\mathrm{H}$, et al. Chronic eosinophilic pneumonia after radiation therapy for breast cancer. Eur Respir J. 2004;23:9-13. [CrossRef] [PubMed] 